\title{
Effects of Structural and Seismic Factors on the Constant-Strength Ductility Spectra Based on NGA-West2 Database
}

\author{
Jinjun Hu $\mathbb{D}^{1},{ }^{1}$ Qinghui Lai $\mathbb{D}^{1},{ }^{1}$ Xuan Liu, ${ }^{2}$ and Lili Xie ${ }^{1}$ \\ ${ }^{1}$ Key Laboratory of Earthquake Engineering and Engineering Vibration, Institute of Engineering Mechanics, \\ China Earthquake Administration, Harbin 150080, China \\ ${ }^{2}$ School of Civil Engineering, Tianjin University/Key Laboratory of Coast Civil Structure Safety of China Ministry of Education, \\ Tianjin University, Tianjin 300072, China \\ Correspondence should be addressed to Jinjun Hu; hu-jinjun@163.com and Qinghui Lai; 18845117968@163.com
}

Received 17 March 2020; Revised 21 June 2020; Accepted 4 July 2020; Published 27 July 2020

Academic Editor: Alba Sofi

Copyright (c) 2020 Jinjun Hu et al. This is an open access article distributed under the Creative Commons Attribution License, which permits unrestricted use, distribution, and reproduction in any medium, provided the original work is properly cited.

\begin{abstract}
The constant-strength ductility spectrum is a nonlinear response spectrum that is commonly used to establish the demand curve of the seismic response during performance-based seismic design. It is affected by many factors. In this paper, to evaluate the effect of the major influencing factors, including the structural parameters and seismic factors, the constant-strength ductility spectrum is calculated under different conditions based on 5535 ground motion records. Conclusions are drawn based on the mean constant-strength ductility spectra. (1) With respect to the effects of structural factors, the variation trend of the ductility spectra is highly consistent with increasing $T$, and the ductility spectra are usually larger for larger $\zeta$ and smaller $C_{y}$ and $k_{2}$. (2) With respect to the seismic factors, the ductility spectra show obvious differences in different periods; however, some parameters, such as PGA, have no influence on the ductility spectra. The results of this study can provide a theoretical basis for the calculation of ductility demand in the seismic design of structures.
\end{abstract}

\section{Introduction}

Nonlinear response spectra are used to establish the demand curves of seismic responses. It is generally accepted that the most accurate method for the seismic performance evaluation and seismic response analysis of structures is the nonlinear time history analysis. In the 1970s, Newmark and Hall [1] proposed the equal energy principle and the equal displacement principle to study the relationship between the yield strength coefficient $\left(C_{y}\right)$ and the displacement ductility demand $(u)$. Subsequently, many experts have performed considerable research on the relationship between $C_{y}$ and $u$ [2-4]. In recent years, many studies have focused on the effects of structural parameters on constant-ductility seismic resistance spectra by a large number of ground motion records [5-9]. Xie and Zhai studied the influence of the restoring force model on constant-ductility seismic resistance spectra by using a large number of ground motion records; they mainly studied the variation in the seismic resistance coefficient under different ductility values, postyield stiffness ratio values of the bilinear model $\left(k_{2}\right)$, and damping ratios $(\zeta)[6,7]$. Similarly, some researchers have studied the effects of structural parameters and seismic factors on the constant-strength response spectra and constant-ductility response spectra [10, 11]. Miranda [12, 13] explained the influencing factors of magnitude, epicentre distance, and other factors on the constant-ductility response spectrum in detail. Yi and Zhang [14] compared the advantages and disadvantages of constant-ductility strength spectra and constant-strength ductility spectra; it was concluded that the method for calculating constant-strength ductility spectra is simpler and does not require iterative calculations. Additionally, only one $u$ value can be obtained in a given period, while more than one value of $C_{y}$ may be applied when the $u$ value is fixed. In addition, Some people made improvements in structural parameters to research inelastic response demand. Michel et al. [15] proposed an improved method to the original EC 8 for displacement 
demand prediction. And the new proposals significantly improve the reliability of displacement demand prediction for values of strength reduction factors greater than 2 compared to the original EC 8 procedure. Castaldo et al. $[16,17]$ aimed to propose seismic reliability-based relationships between the behavior factors and the displacement demand for nonlinear hardening and softening structures isolated by friction pendulum system devices and double concave sliding bearings. There are some researchers also studied the effects of different types of strong motions on constant-strength response spectra such as mainshock-aftershock sequence ground motion and pulse-like ground motions [18-20].

The above studies discussed the influence of some factors on constant-strength ductility spectra. But very few research studies have analyzed amplitude, duration, and frequency spectra parameters comprehensively, which is the most important parameters reflecting the characteristics of ground motion. Zhai and Li [21] studied the effects on the nonlinear displacement ratio spectra under various ground parameters such as PGA and PGV. But the effects of these factors on the constant-strength ductility spectrum were not analyzed.

Therefore, this paper conducted a comprehensive analysis of the factors that affect constant-strength ductility spectra. These factors include structural parameters and seismic parameters. The modified Clough model [22] is selected for calculating the constant-strength ductility spectra. The variation rule of constant-strength ductility spectra can be obtained under different structural parameters and seismic factors. By changing the factors, the constant-strength ductility spectra will change, and the variation rule can be analyzed.

\section{Ground Motion and SDOF Structural Systems}

2.1. Ground Motions. The ground motion records are selected from the NGA-West2 database released by the Pacific Earthquake Engineering Research Center (PEER), which includes 21,540 groups of three-component ground motion records from 1935 to 2011 [23]. This paper mainly focuses on strong ground motions that are destructive to structures. Therefore, horizontal component ground motion records with peak ground accelerations (PGAs) greater than $50 \mathrm{gal}$ are selected. The selected dataset includes 5,535 ground motion records. The space distribution of ground motion records is shown in Figure 1, and it can be concluded that the selected ground motion records are mainly distributed in the Mediterranean and the Pacific Coast regions. The magnitude-epicentre distance distribution is shown in Figure 2.

2.2. Structural Systems. There are many kinds of structural hysteretic model such as elastic perfectly plastic (EPP) model, modified Clough (MC) model, and pinching model $[18,24]$. The modified Clough model as shown in Figure 3 is selected with reference to Clough's study [25] for calculating the constant-strength ductility spectra in this paper. And it was used to simulate the flexural behavior that exhibits stiffness degradation at reloading. After the nonlinear response of the structure, the loading stiffness of the structure will gradually decrease with the reloading process. As shown in Figure 3, $\mathrm{K}_{0}$ is the initial stiffness and other parameters will be described in detail later.

The seismic displacement ductility was chosen in this study as the parameter representative of damage due to large deformations. The definition of the displacement ductility $u$ is relatively uniform. Most researches adopted the following definition [18-20]:

$$
u=\frac{u_{m}}{u_{y}}
$$

where $u_{m}$ is the maximum displacement response of the SDOF system under ground motions and $u_{y}$ is the yield displacement of the structure.

The second structural parameter is the yield strength coefficient $\left(C_{y}\right)$, which is defined as

$$
C_{y}=\frac{f_{y}}{f_{e}}
$$

where $f_{e}$ is the strength demand on an infinitely elastic SDOF system, and $f_{y}$ is the yield strength of the corresponding inelastic SDOF system with the same mass and initial stiffness. Four values of $C_{y}\left(C_{y}=0.3,0.5,0.7\right.$, and 0.9$)$ are considered to examine the influence of the structural strength.

Structural parameters also include the postyield stiffness ratio $\left(k_{2}\right)$ and damping ratio $(\zeta)$ and $k_{2}$ is the ratio of the postyield stiffness to the initial stiffness in the hysteretic model. And $\zeta$ is defined as

$$
\xi=\frac{c}{2 m w},
$$

where $c$ is damping coefficient and $w$ and $m$ are the natural frequency and mass of the system, respectively. In practical engineering, the damping ratio is usually 0.05 .

\section{Structural and Seismic Factors}

To better understand the influence of strong motion records on the constant-strength ductility spectra, it is necessary to identify the various effects of the most important factors. The uncertainty of structural response under strong earthquake mainly comes from two aspects: (a) structural uncertainty and (b) uncertainty of ground motions. Therefore, this paper mainly analyzes these effects in terms of structural parameters and seismic factors. The detailed influencing factors are shown in Table 1.

\section{Effect of Structural Parameters on the Constant-Strength Ductility Spectra}

The structural parameters of a SDOF system have certain influence on the structural response during an earthquake. The structural parameters mainly include $T, C_{y}, \zeta$, and $k_{2}$. Some researchers have studied the influence of structural parameters on the constant-strength ductility spectra $[14,20,26]$. When the structural parameters change, the 


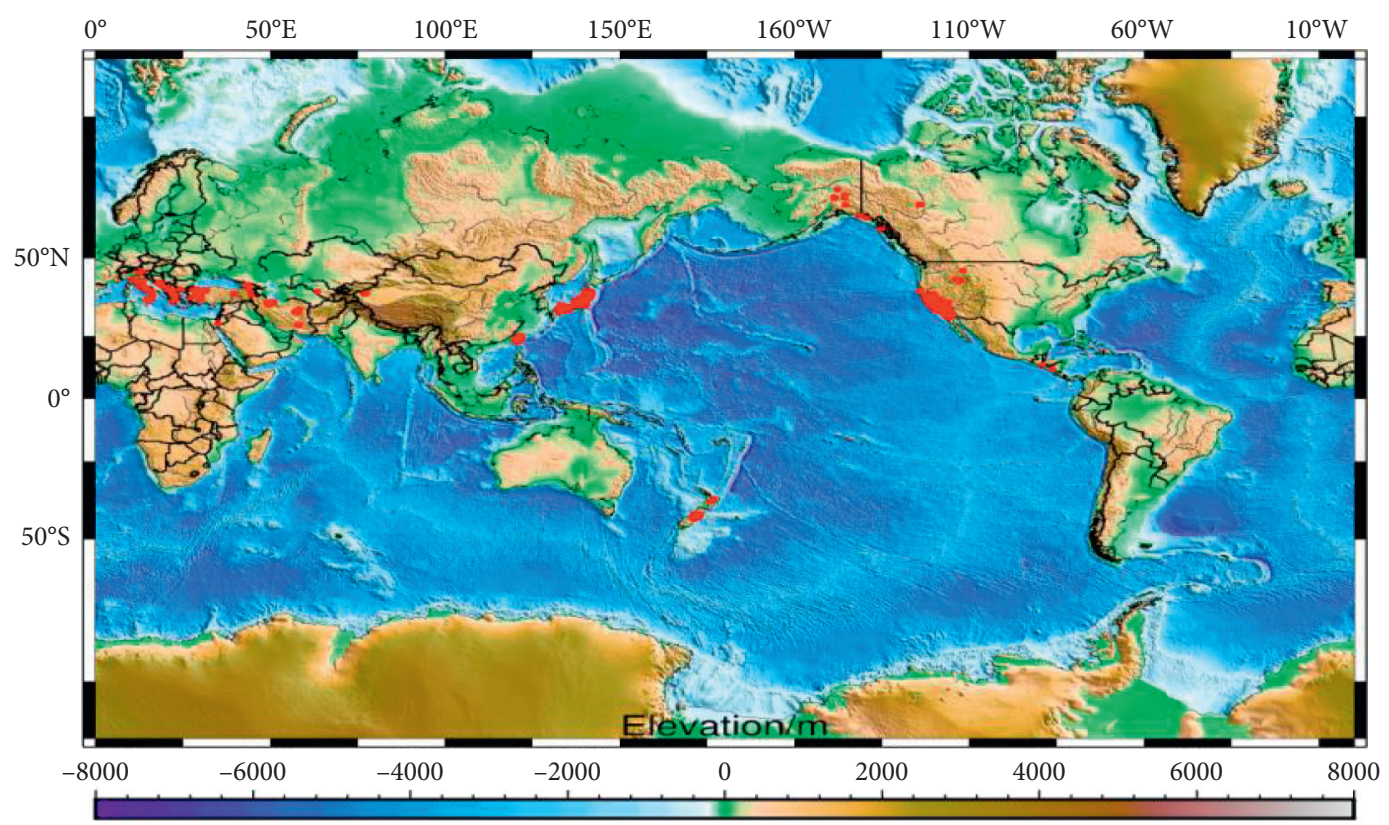

Figure 1: Data distribution of the selected ground motion records.

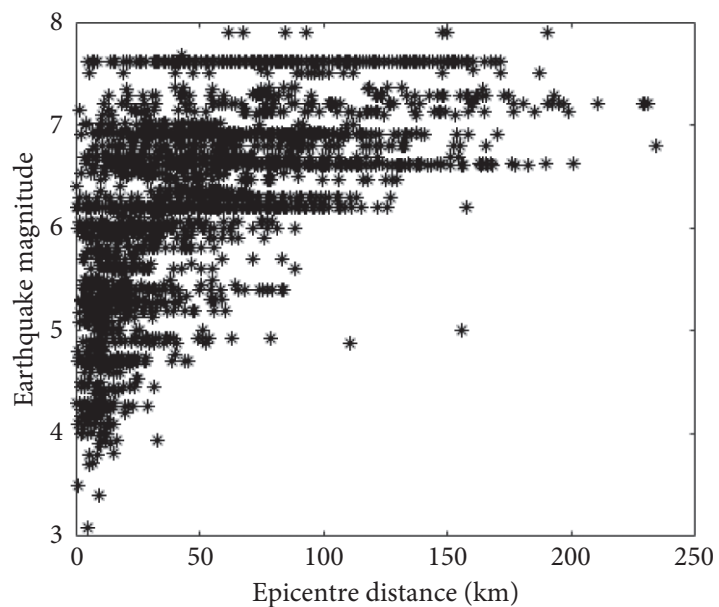

FIgURE 2: Magnitude-epicentre distance distribution of the selected ground motion records.

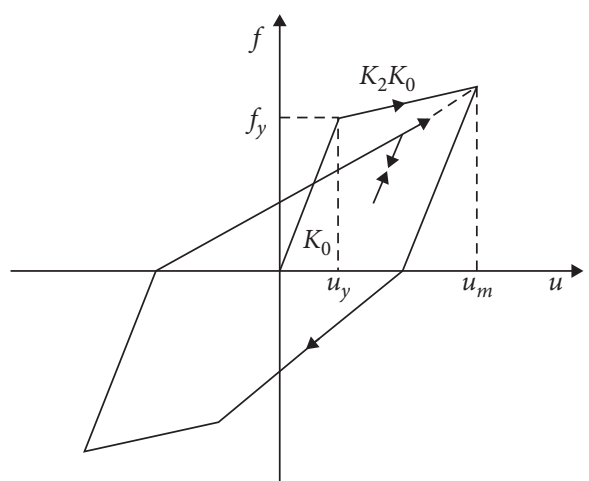

Figure 3: The modified Clough model.
TABLE 1: Influencing factors and parameters.

\begin{tabular}{lc}
\hline Different aspects & Parameters \\
\hline Structural parameters & Period $(T)$ \\
& The yield strength coefficient $\left(C_{y}\right)$ \\
Damping ratio $(\zeta)$ \\
The postyield stiffness ratio $\left(k_{2}\right)$ \\
Earthquake magnitude $(M)$ \\
Epicentre distance $(R)$ \\
Seismic factors & Peak ground acceleration $(\mathrm{PGA})$ \\
& Effective peak velocity $(\mathrm{EPV})$ \\
& Significant duration $\left(D_{s}\right)$ \\
\hline
\end{tabular}

constant-strength ductility spectra change accordingly. Therefore, according to the existing research results, this paper explains the effect of these parameters on the constantstrength ductility spectra.

The constant-strength ductility spectra are calculated as shown in Figure 4 based on 5535 ground motion records under specific conditions: $C_{y}=0.3, \zeta=0.05, k_{2}=0$. It can be seen from Figure 4 that the trend of the mean constantstrength ductility spectra is consistent with that of all constant-strength ductility spectra. Therefore, when explaining the influence of different factors on the constantstrength ductility spectra, we mainly study the effect of different factors on the mean constant-strength ductility spectra.

4.1. Effect of the Period and the Yield Strength Coefficient. To study the influence of $T$ and $C_{y}$ on the constant-strength ductility spectra, the mean constant-strength ductility spectra under different $C_{y}$ values is calculated when $\zeta$ is 0.05 and $k_{2}$ is 0 , as shown in Figure 5. Four values of $C_{y}$ are considered $\left(C_{y}=0.3,0.5,0.7\right.$, and 0.9$)$, and the following conclusions are drawn from the analysis. 


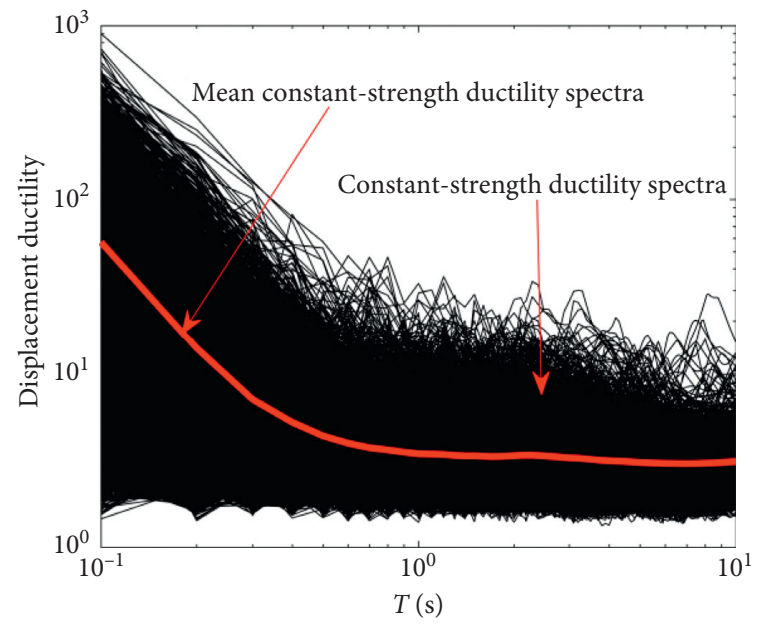

FIgURE 4: Constant-strength ductility spectra of selected ground motion records.

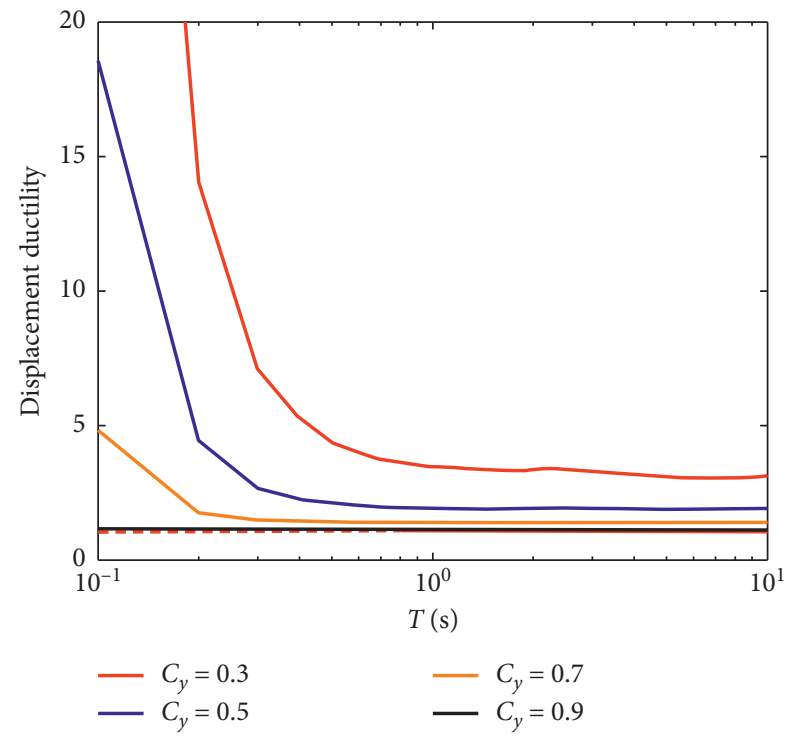

Figure 5: Effect of different yield strength coefficients $\left(C_{y}=0.3,0.5\right.$, 0.7 , and 0.9 ) on the variation of the mean constant-strength ductility spectra.

For constant period, the smaller the value of $C_{y}$, the larger the values of $u$. For constant $C_{y}$, the values of $u$ decrease fast with the increase of the $T$ in short-period section. The values of $u$ do not change significantly with the period in the medium- and long-period ranges, particularly for bigger $C_{y}$ values, and thus the constant-strength ductility spectra can be characterized as period independent. This variation rule is the same as the result of $\mathrm{Li}$ and Yi's research $[14,20]$.

4.2. Effect of the Damping Ratio. To analyse the influence of $\zeta$ on the constant-strength ductility spectra, the variation in the mean constant-strength ductility spectra under different $\zeta$ values is calculated when $C_{y}$ is 0.3 and $k_{2}$ is 0 . The variation is shown in Figure 6. Four values of $\zeta(\zeta=0.01,0.05,0.1$, and $0.15)$ are considered. The following conclusions are drawn from the analysis.

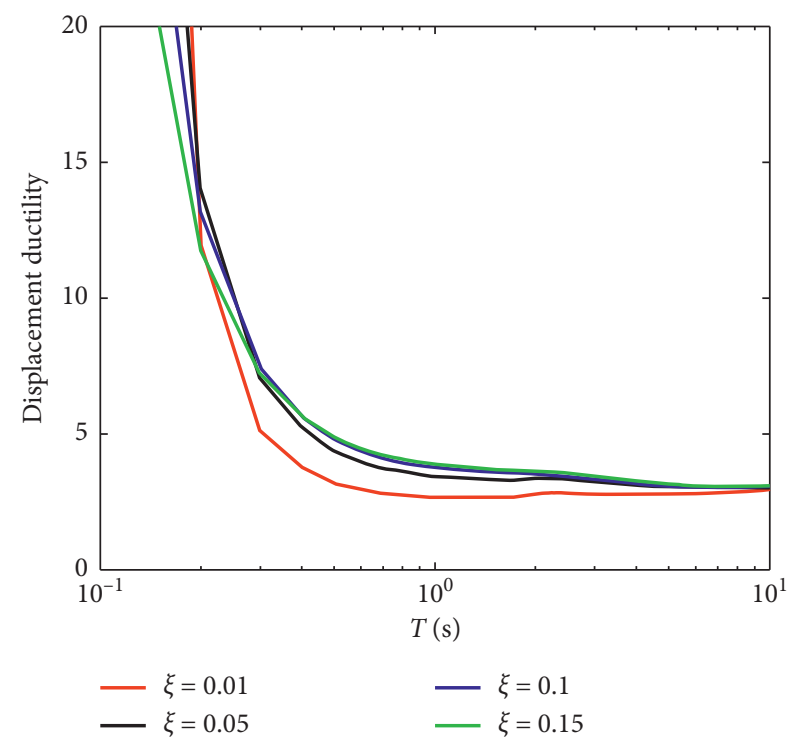

FIGURE 6: Effect of $\zeta(\zeta=0.01,0.05,0.1$, and 0.15$)$ on the variation of the mean constant-strength ductility spectra.

In short-period range, the variation rule of the constantstrength ductility spectra is complex. However, in mediumand long-period ranges. When $\zeta$ changes, the displacement ductility value changes obviously, and the smaller the value of $\zeta$ is, the slower the value of $u$ decreases. Additionally, the rate of decrease of $u$ varies based on the damping ratio. Therefore, the effect of $\zeta$ on the constant-strength ductility spectra cannot be neglected.

4.3. Effect of the Postyield Stiffness Ratio of the Bilinear Model. To quantitatively analyse the effect of $k_{2}$ on the constantstrength ductility spectra, the variation in the constantstrength ductility spectra under different $k_{2}$ conditions is calculated when $C_{y}$ is 0.3 and $\zeta$ is 0.05 . Four values of $k_{2}$ $\left(k_{2}=0,0.05,0.1\right.$, and 0.15$)$ are considered. The variation is shown in Figure 7. The following conclusions are drawn from the analysis.

The variation rule of the constant-strength ductility spectra is obviously consistent at all periods. For different $k_{2}$ values, with the increase in $k_{2}$, the constant-strength ductility spectra values gradually decrease for a constant period.

\section{Effect of Seismic Factors on the Constant- Ductility Strength Spectra}

Seismic factors mainly include the earthquake magnitude, epicentre distance, and ground motion parameters. These factors also influence the constant-strength ductility spectra value. Before analyzing the influence of seismic factors on the constant-strength ductility spectra, the ground motion records are classified according to the site type, and the structure parameters are defined as follows: (1) $C_{y}=0.3$; (2) $\zeta=0.05$; (3) $k_{2}=0$.

Using the USGS site classification method, 5535 ground motion records are classified into five site types, and the 


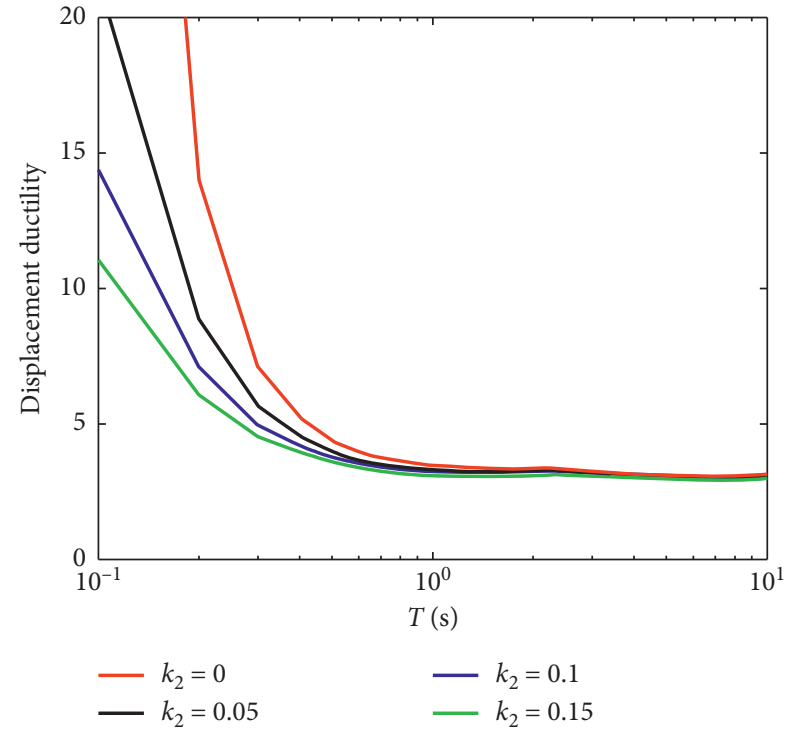

FIGURE 7: Effect of $k_{2}\left(k_{2}=0,0.05,0.1\right.$, and 0.15$)$ on the variation of the mean constant-strength ductility spectra.

classification result is shown in Table 2. According to the classification results, the number of ground motion records varies greatly by site type, and some sites have very few ground motion records. To eliminate the influence of the number of ground motion records on the constant-strength ductility spectra, site A type, site B type, and site $C$ type are combined as stiff soil site, and site D type and site E type are combined as soft soil site. The earthquake magnitude and epicentre distance distribution of records in different site classifications are shown in Figure 8.

5.1. Effect of Earthquake Magnitude. To analyze the effect of earthquake magnitude $M$ on constant-strength ductility spectra, the ground motion records are divided into four types according to earthquake magnitude, that is, $M<5$, $5 \leq M<6,6 \leq M<7$, and $M \geq 7$, and the classification results are shown in Table 3 . The mean constant-strength ductility spectra are calculated in each type, and the variation rule of the mean constant-strength ductility spectra with different magnitudes is analyzed as shown in Figure 9. The following conclusions can be drawn from the analysis.

The mean constant-strength ductility spectra show obvious differences for different earthquake magnitudes. For the results in stiff soil site, in short and medium ranges, the greater the magnitude, the larger the ductility spectra value. In long-period range, the ductility spectra values of different magnitudes overlap, and there is no obvious variation. For the results in soft soil sites, in short and medium ranges, there is a variation similar to that of the ductility spectra values in stiff soil sites. However, in long-period range, the larger the magnitude, the smaller the ductility spectra value.

5.2. Effect of Epicentre Distance. To analyze the influence of the epicentre distance on the constant-strength ductility spectra, the ground motion records of every site type are divided into two groups according to epicentre distance: (1) near-field; (2) far-field. The classification result is shown in Table 4. The variation rule of the mean constantstrength ductility spectra with different epicentre distances is analyzed in stiff and soft soil sites, as shown in Figure 10. The following conclusions can be drawn from the analysis.

The mean constant-strength ductility spectra of the different types show obvious differences. For ground motions in stiff soil, in short- and medium-period ranges, the farther the epicentre distance, the larger the ductility spectra value. In contrast, in long-period ranges, the ductility spectra value is usually smaller in the near-field than in far-field. And this phenomenon is more obvious in soft soil sites.

5.3. Effect of Ground Motion Parameters on Constant-Ductility Strength Spectra. The nonlinear responses of structures are closely related to the ground motion parameters. To explain the effects of different ground motion parameters on constant-strength ductility spectra, the representative parameters of amplitude, frequency spectra, and duration are chosen to analyse the variation of constant-strength ductility spectra in this paper. Therefore, in this paper, the representative parameters of amplitude, frequency spectra, and duration are chosen to analyse the variation of constantstrength ductility spectra. The definitions of the structural parameters are the same as those in the previous section.

The selected representative ground motion parameters are the peak ground acceleration (PGA), the effective peak velocity $(\mathrm{EPV})$, and the significant duration $\left(D_{s 5-95}\right)$. For seismic structural analysis purposes, the most simple and widely used intensity index is PGA, and it is directly obtained from the ground motion record itself. The calculation of EPV is shown as follows:

$$
\mathrm{EPV}=\frac{S_{v}}{2.5}
$$

where $S_{v}$ is the mean velocity response spectrum with a damping ratio of 0.05 near the period of 1 second, and the period range is usually from 0.8 to 1.2 seconds.

For the $D_{s 5-95}$, Husid [27] proposed an intensity parameter that indicates the increase of ground motion energy over time. Its standardized form is shown as follows:

$$
I(t)=\frac{\int_{0}^{t} a^{2}(t) \mathrm{d} t}{\int_{0}^{T} a^{2}(t) \mathrm{d} t}
$$

where the $T$ is the total duration of ground motion and $I(t)$ is a function from 0 1. Trifunac and Brady [28] defined a significant duration of ground motion as follows:

$$
\left\{\begin{array}{l}
I\left(T_{1}\right)=0.05, \\
I\left(T_{2}\right)=0.95, \\
D_{s 5-95}=T_{2}-T_{1},
\end{array}\right.
$$

where $T_{1}$ and $T_{2}$ are, respectively, the times where $5 \%$ and $95 \%$ of ground motion energy are reached. The significant 
TABLE 2: Classification results of ground motion records based on USGS site classification.

\begin{tabular}{lccc}
\hline Site type & Classification standard Vs30 $(\mathrm{m} / \mathrm{s})$ & Number 1 & Site type \\
\hline Site A type & $>1500$ & 7 & Number 2 \\
Site B type & $760 \sim 1500$ & 101 & Stiff soil \\
Site C type & $360 \sim 760$ & 2742 & \multirow{2}{*}{ Soft soil } \\
Site D type & $180 \sim 360$ & 2533 & 152 \\
Site E type & $<180$ & 150 & 2685 \\
\hline
\end{tabular}

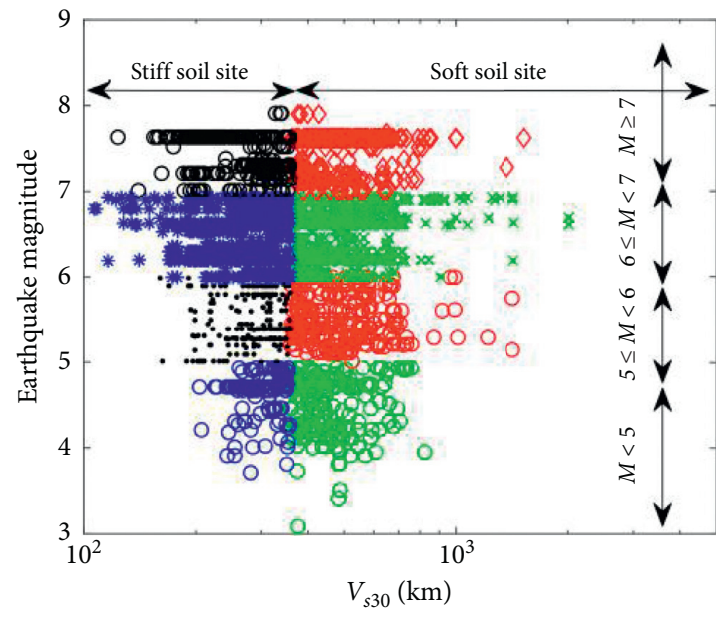

(a)

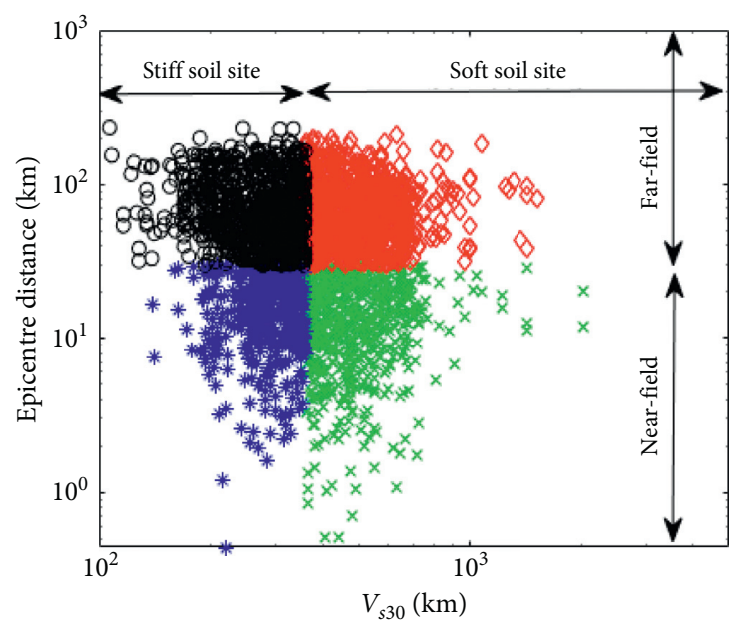

(b)

Figure 8: (a) Earthquake magnitude- $V_{s 30}$ distribution and (b) epicentre distance- $V_{s 30}$ distribution in different site classifications.

TABLE 3: Classification results of ground motion records based on earthquake magnitude.

\begin{tabular}{lcccc}
\hline Site type & $M<5$ & $5 \leq M<6$ & $6 \leq M<7$ & $M \geq 7$ \\
\hline Stiff soil site & 301 & 556 & 1441 & 552 \\
Soft soil site & 226 & 589 & 1323 & 547 \\
\hline
\end{tabular}

duration $D_{\text {s5-95 }}$ as defined in equation (6) is most widely used for evaluating the effective duration of earthquakes.

According to these parameters, 5535 ground motion records are classified. The classification results are shown in Table 5, and the PGA-EPV and PGA-D ${ }_{\text {s5-90 }}$ distribution of ground motion records are shown in Figure 11.

5.3.1. Effect of PGA. To study the effect of PGA on the constant-strength ductility spectra, the ground motions are divided into two categories: (1) PGA > 200 gal and (2) $\mathrm{PGA} \leq 200$ gal. The variation of the mean constant-strength ductility spectra is analyzed, and the results are shown in Figure 12. It can be concluded that PGA has little effect on the mean constant-strength ductility spectra values at all periods.

5.3.2. Effect of EPV. To study the effect of EPV on the constant-strength ductility spectra, the ground motion records are divided into two categories: (1) EPV $\leq 10 \mathrm{~cm} / \mathrm{s}$ and (2) EPV $>10 \mathrm{~cm} / \mathrm{s}$. The variation of the mean constantstrength ductility spectra is analyzed, and the results are shown in Figure 13. It can be concluded that EPV has a significant effect on the mean constant-strength ductility spectra values in short-period range; the larger the EPV of the ground motion record, the larger the ductility spectra. But in the medium- and long-period ranges, EPV has little effect on the ductility spectra values.

5.3.3. Effect of $D_{s 5-95}$. To analyze the influence of $D_{s 5-95}$ on the constant-strength ductility spectra, the ground motions are divided into two categories: (1) $D_{s 5-95} \leq 25 \mathrm{~s}$ and (2) $D_{s 5-95}>25 \mathrm{~s}$. The variation of the mean constant-strength ductility spectra is analyzed, and the results are shown in Figure 14. In the long-period range, long-duration ground motion records usually have larger spectra values than shortduration ground motion records. But in short- and mediumperiod ranges, the longer the duration of ground motion, the larger the ductility spectra. 


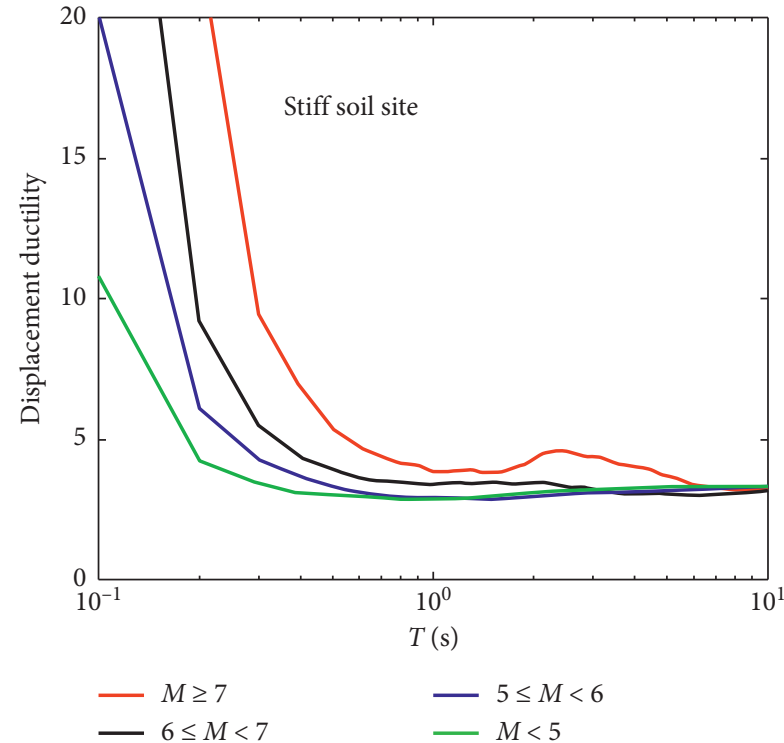

(a)
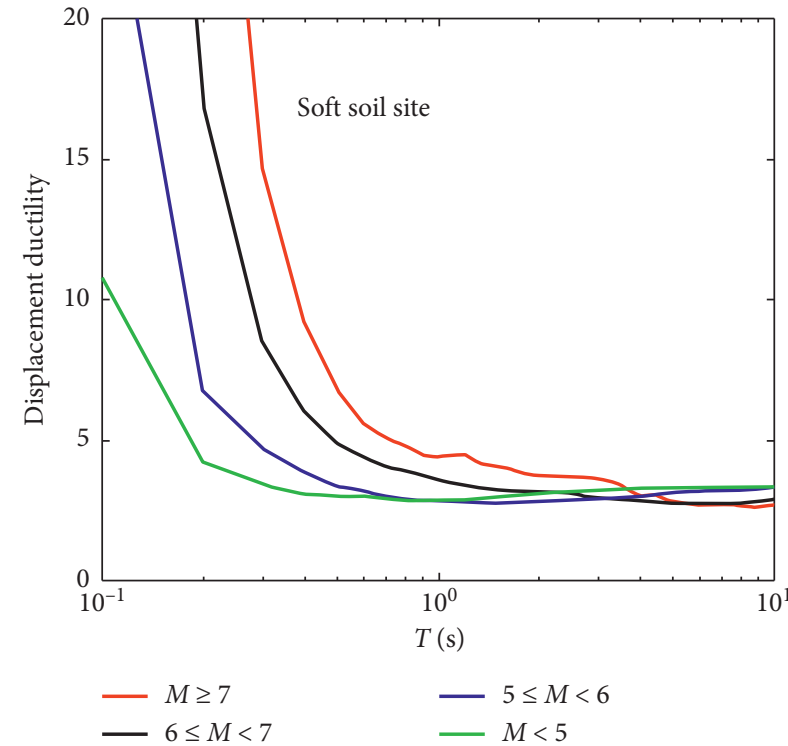

(b)

FIGURE 9: Effect of earthquake magnitude on the variation of mean constant-strength ductility spectra: (a) stiff soil site; (b) soft soil site.

TABLE 4: Classification results of ground motion records based on epicentre distance.

\begin{tabular}{lccc}
\hline Site type & Type & Epicentre distance $(\mathrm{km})$ & Numbers \\
\hline \multirow{2}{*}{ Stiff soil } & Near-field & $<30$ & 1227 \\
& Far-field & $>30$ & 1623 \\
Soft soil & Near-field & $<30$ & 936 \\
& Far-field & $>30$ & 1749 \\
\hline
\end{tabular}

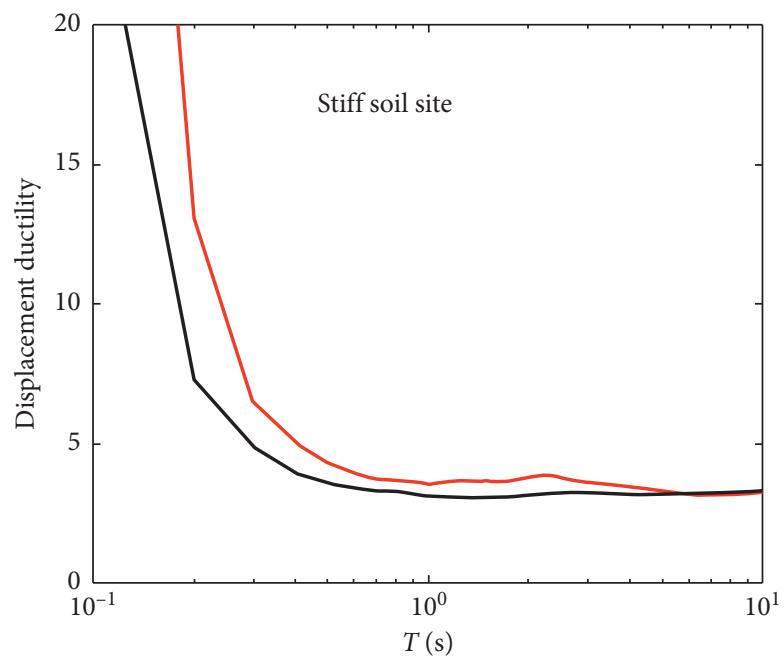

- Far-field

— Near-field

(a)

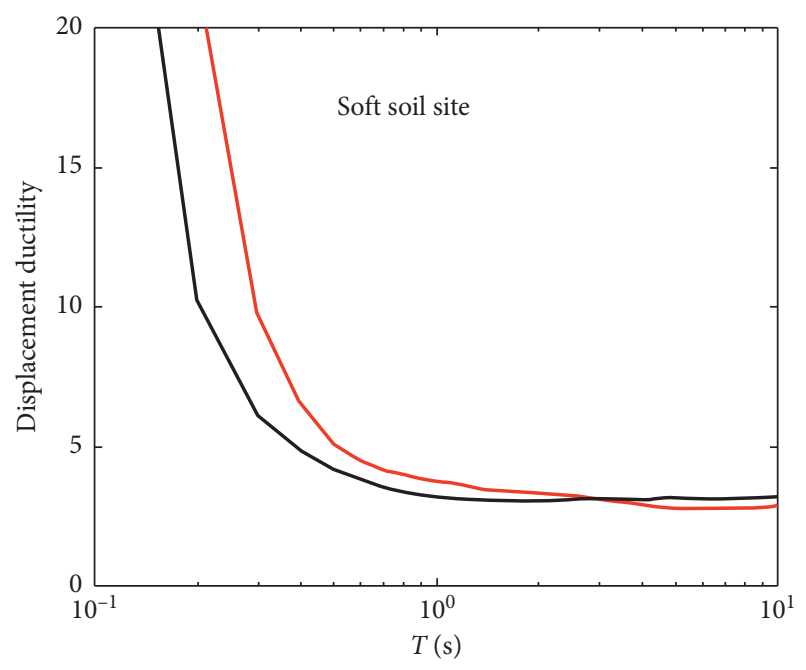

Far-field

- Near-field

FIGURE 10: Effect of epicentre distance on the variation of mean constant-strength ductility spectra: (a) stiff soil site; (b) soft soil site. 
TABLE 5: Classification results of ground motion records based on ground motion parameters.

\begin{tabular}{lcc}
\hline Ground motion parameters & Range of values & Numbers \\
\hline \multirow{2}{*}{ PGA } & $\leq 200 \mathrm{gal}$ & 4296 \\
& $>200 \mathrm{gal}$ & 1239 \\
EPV & $\leq 10 \mathrm{~cm} / \mathrm{s}$ & 3728 \\
& $>10 \mathrm{~cm} / \mathrm{s}$ & 1807 \\
$D_{\text {s5-95 }}$ & $\leq 25 \mathrm{~s}$ & 4456 \\
\end{tabular}

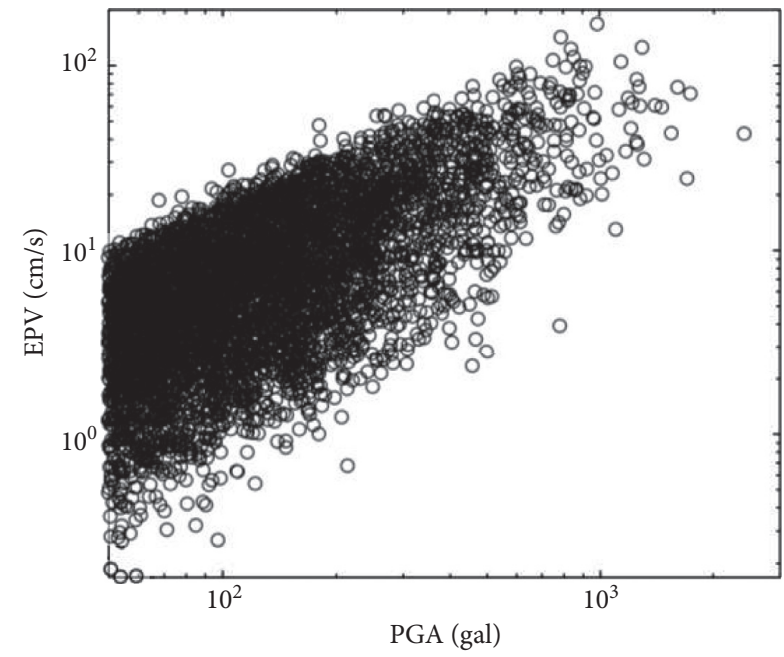

(a)

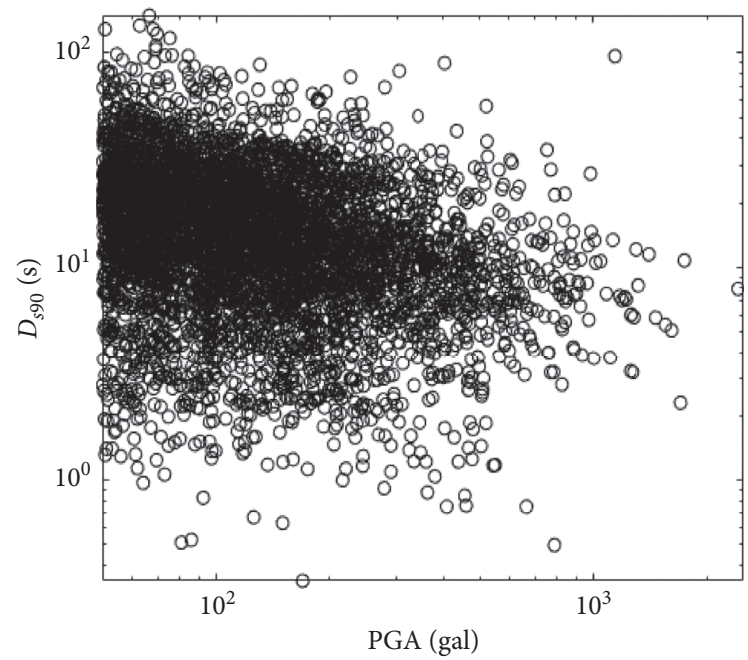

(b)

Figure 11: (a) PGA-EPV distribution and (b) PGA- $D_{s 5-90}$ distribution of selected ground motion records.

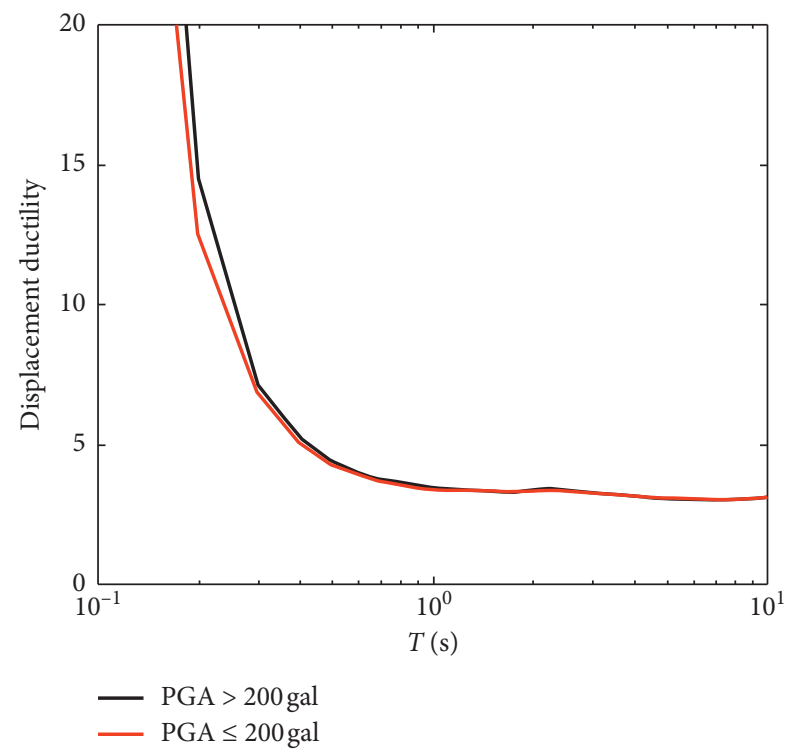

FIGURE 12: Effect of PGA on the variation of mean constantstrength ductility spectra.

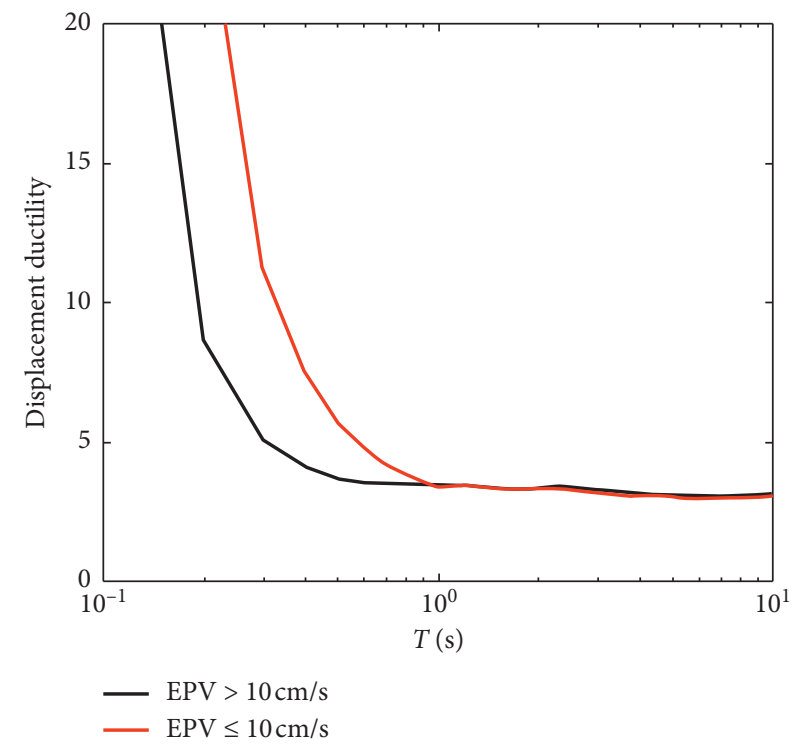

FIGURE 13: Effect of EPV on the variation of mean constantstrength ductility spectra. 


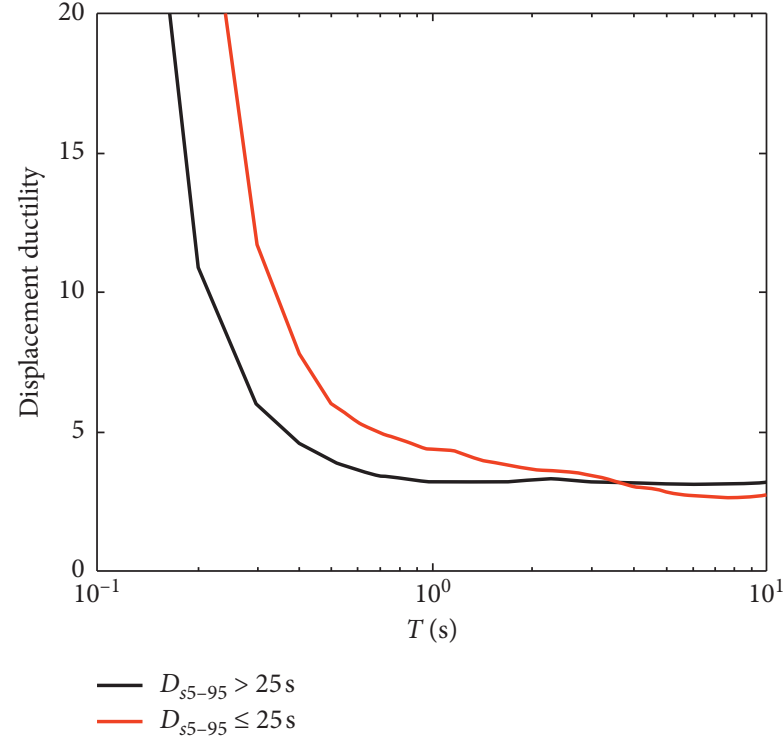

Figure 14: Effect of $D_{s 5-95}$ on the variation of the mean constantstrength ductility spectra.

\section{Conclusion and Discussion}

This study compares the influences of different factors on the constant-strength ductility spectra based on the 5535 ground motion records in NGA-West2 database, and the effects of structural parameters and seismic factors on the spectra are analyzed. The following conclusions are inferred from the results of this study:

(1) With respect to the effects of structural parameters, except for $\zeta$ at short-period ranges, the variation of the ductility spectra value is highly consistent with the increasing $T$. And the ductility spectra values are usually larger for larger $\zeta$ and smaller $C_{y}$ and $k_{2}$.

(2) With respect to the effects of seismic factors, the ductility spectra values show obvious differences in different period ranges. In short-period range, when the epicentre distance and magnitude of the ground motion records are larger, the corresponding ductility demand is higher. However, for EPV and $D_{s 5-95}$, when the EPV and $D_{s 5-95}$ of the ground motion records are larger, the corresponding ductility demand is small. In the medium- and longperiod ranges, the ductility spectra value varies under different conditions; however, some parameters, such as EPV and PGA, have little effect on the ductility spectra values.

As one of the nonlinear response spectra, the constantstrength ductility demand spectra are affected by many factors; in this paper, we studied as many influencing factors as possible from the structural and seismic aspects. The effect of a portion of factors studied in this paper are compared with the results of the former studies, while the effect of some other factors were not compared due to lack of analysis of these factors in the previous studies [14, 20]. So it is still necessary to compare the results with the others in the future. In conclusion, the results of this study provide a statistical and quantitative basis for the calculation of ductility demand in the seismic design of structures.

\section{Data Availability}

Strong motion records were provided by the Pacific Earthquake Engineering Research Center (PEER).

\section{Conflicts of Interest}

The authors declare that they have no conflicts of interest.

\section{Acknowledgments}

This work was supported by the Scientific Research Fund of the Institute of Engineering Mechanics, China Earthquake Administration (Grant no. 2019A01). and the National Natural Science Foundation of China (Grant no. U1939210). The authors also appreciate the assistance of the Pacific Earthquake Engineering Research Center (PEER) in providing the strong motion records.

\section{References}

[1] N. M. Newmark and W. J. Hall, "Seismic design criteria and nuclear reactor facilities," Building Practices for Disaster Mitigation, vol. 46, pp. 209-236, National Bureau of Standard, U.S. Department of Commerce, Gaithersburg, MD, USA, 1973.

[2] A. A. Nassar and H. Krawinkler, "Seismic demands for SDOF and MDOF systems. Report no. 95," The John A. Blume Earthquake Engineering Center, Stanford University, vol. 95, no. 4, , pp. 12-45, Stanford, CA, USA, 1991.

[3] S. Pal, S. S. Dasaka, and A. K. Jain, "Inelastic response spectra," Computers \& Structures, vol. 25, no. 3, pp. 335-344, 1987.

[4] T. Vidic, P. Fajfar, and M. Fischinger, "Consistent inelastic design spectra: strength and displacement," Earthquake Engineering \& Structural Dynamics, vol. 23, no. 5, pp. 507-521, 1994.

[5] B.-F. Peng and J. P. Conte, "Statistical insight into constantductility design using a non-stationary earthquake ground motion model," Earthquake Engineering \& Structural Dynamics, vol. 26, no. 9, pp. 895-916, 1997.

[6] L.-1. Xie and C.-h. Zhai, "Study on the severest real ground motion for seismic design and analysis," Acta Seismologica Sinica, vol. 16, no. 3, pp. 260-271, 2003.

[7] C. H. Zhai, L. L. Xie, and M. H. Zhang, "Influence of damping on the spectra of constant-ductility seismic resistance of engineering structures," Journal of Harbin Institute of Technology, vol. 38, no. 10, pp. 1705-1708, 2006, in Chinese.

[8] S. L. Xue, Z. Yu, and G. Xu, "Elastoplastic dynamic time history analysis of Yinchuan De Feng building," Building Structure, vol. 47, no. 5, pp. 37-42, 2017, in Chinese.

[9] H. j He, M. H. Xu, and L. Su, "Study on the number of input ground motion in elastoplastic time history analysis of structures," Earthquake Engineering and Engineering Vibration, vol. 38, no. 2, pp. 150-156, 2018, in Chinese.

[10] D. S. Zhou and X. L. Lu, "Ducitility demand spectra and inelastic displacement spectra considering soil conditions and design characteristic periods," Earthquake Engineering and Engineering Vibration, vol. 24, no. 1, pp. 39-48, 2004, in Chinese.

[11] R. Rupakhety and R. Sigbjörnsson, "Ground-Motion Prediction Equations (GMPEs) for inelastic displacement and 
ductility demands of constant-strength SDOF systems," Bulletin of Earthquake Engineering, vol. 7, no. 3, pp. 661-679, 2009.

[12] E. Miranda and V. V. Bertero, "Evaluation of strength reduction factors for earthquake-resistant design," Earthquake Spectra, vol. 10, no. 2, pp. 357-379, 1994.

[13] E. Miranda, "Inelastic Displacement ratios for structures on firm sites," Journal of Structural Engineering, vol. 126, no. 10, pp. 1150-1159, 2000.

[14] W.-J. Yi, H.-Y. Zhang, and S. K. Kunnath, "Probabilistic constant-strength ductility demand spectra," Journal of Structural Engineering, vol. 133, no. 4, pp. 567-575, 2007.

[15] C. Michel, P. Lestuzzi, and C. Lacave, "Simplified non-linear seismic displacement demand prediction for low period structures," Bulletin of Earthquake Engineering, vol. 12, no. 4, pp. 1563-1581, 2014.

[16] P. Castaldo, B. Palazzo, G. Alfano, and M. F. Palumbo, "Seismic reliability-based ductility demand for hardening and softening structures isolated by friction pendulum bearings," Structural Control and Health Monitoring, vol. 25, no. 11, p. e2256, 2018.

[17] P. Castaldo and G. Alfano, "Seismic reliability-based design of hardening and softening structures isolated by double concave sliding devices," Soil Dynamics and Earthquake Engineering, vol. 129, p. 105930, 2020.

[18] C.-H. Zhai, W.-P. Wen, S. Li, Z. Chen, Z. Chang, and L.-L. Xie, "The damage investigation of inelastic SDOF structure under the mainshock-aftershock sequence-type ground motions," Soil Dynamics and Earthquake Engineering, vol. 59, pp. 30-41, 2014.

[19] C. Zhai, D. Ji, W. Wen, W. Lei, and L. Xie, "Constant ductility energy factors for the near-fault pulse-like ground motions," Journal of Earthquake Engineering, vol. 21, no. 2, pp. 343-358, 2017.

[20] C. Li, S. Kunnath, and C. Zhai, "Influence of early-arriving pulse-like ground motions on ductility demands of singledegree-of-freedom systems," Journal of Earthquake Engineering, vol. 22, no. 9, pp. 1-24, 2018.

[21] C. Zhai, S. Li, and Y. Sun, "Study on inelastic displacement ratio spectra for near-fault pulse-type ground motions," Earthquake Engineering and Engineering Vibration, vol. 6, no. 4, pp. 351-355, 2007.

[22] A. K. Chopra, Dynamics of Structures, Pearson Education Inc., Hoboken, NJ, USA, 5th edition, 2017.

[23] D. A. Timothy, B. D. Robert, and P. S. Jonathan, "NGA-West2 Database," Earthquake Spectra, vol. 30, no. 3, pp. 989-1005, 2014.

[24] E. Miranda and J. Ruiz-Garcia, "Influence of stiffness degradation on strength demands of structures built on soft soil sites," Engineering Structures, vol. 24, no. 10, pp. 1271-1281, 2002.

[25] R. W. Clough and J. Penzien, Dynamics of Structures, McGraw-Hill, New York, NY, USA, 1975.

[26] Q. X. Shi and J. J. Men, "Ductility spectra method with constant $\zeta_{-}$y inperformance-based seismic evaluation of building structures," Earthquake Engineering and Engineering Vibration, vol. 27, no. 1, pp. 54-58, 2007, in Chinese.

[27] R. L. Husid, "Analisis de terremotos:analisis general," Revista del ID1EM, Santiago Chile, vol. 8, no. 1, pp. 21-42, 1969.

[28] M. D. Trifunac and A. G. Brady, "A study on the duration of strong earthquake ground motion," Bulletin of the Seismological Society of America, vol. 65, no. 3, pp. 581-626, 1975. 